\title{
Performance of passive Direct Methanol Fuel Cell: modelling and experimental studies
}

\author{
Beatriz A. Berns ${ }^{1}$, Mariana F. Torres ${ }^{2}$, Vânia B. Oliveira ${ }^{1 a}$, Alexandra \\ M. F. R. Pinto ${ }^{1 b}$ \\ ${ }^{1}$ CEFT, Department of Chemical Engineering, Faculty of Engineering, University of Porto, Porto, \\ Portugal; 1avaniaso@fe.up.pt, 1bapinto@fe.up.pt; 2Department of Chemical Engineering, \\ Faculty of Engineering, University of Porto, Porto, Portugal.
}

\begin{abstract}
Low methanol and water crossover with high methanol concentrations are essential requirements for a passive Direct Methanol Fuel Cell (DMFC) to be used in portable applications. Therefore, it is extremely important to clearly understand and study the effect of the different operating and configuration parameters on the cell's performance and both methanol and water crossover. In the present work, a detailed experimental study on the performance of an in-house developed passive DMFC with $25 \mathrm{~cm}^{2}$ of active membrane area is described. Tailored membrane electrode assemblies (MEAs) with different structures and combinations of gas diffusion layers (GDL) and membranes, were tested in order to select optimal working conditions at high methanol concentration levels without sacrificing performance. The experimental polarization curves were successfully compared with the predictions of a steady state, one-dimensional model accounting for coupled heat and mass transfer, along with the electrochemical reactions occurring in the passive DMFC developed by the same authors.
\end{abstract}

Author Keywords. Passive direct methanol fuel cells, methanol crossover, net water transport coefficient, simulation, experimental studies.

\section{Introduction}

In the past few years, energy needs for portable electronic devices are rising rapidly due to the increasing amount of applications, especially cell phones. Nowadays most cell phones incorporate energy consuming functionalities like graphics, games, internet service, instant messaging and cameras. Conventional batteries are soon becoming inadequate for the increasing power demand and complexity of portable electronics devices. The time these portable devices can operate as truly portable is limited by the quantity of energy that can be stored within the batteries.

Fuel cell systems and particularly direct methanol fuel cells (DMFC) are considered as a possible solution to replace today's batteries as the dominant power provider for portable applications (Barbir 2005, Sundmacher 2010, Pan 2006). The DMFC are capable of replacing the conventional batteries due to their high energy density and inherent simplicity of operation with methanol as the liquid fuel. This system is more performant, less expensive, environmentally safer, much more efficient and can be used either in a plane, train, car or in remote areas where there is no electricity. The product is cost competitive due to the large market size and economies of scale.

The fuel and oxidant can be supplied to a DMFC in an active or a passive way. In the passive feed system, the fuel pump and air blower are eliminated. The fuel is supplied to the anode from a fuel reservoir built-in in the anode and the air to the cathode, normally by natural 
convection. The refueling of the passive DMFC is fast and the fuel can last several months. However, this simple design causes lower system performance due to the difficulty of getting a continuous and homogeneous supply of reactants to the anode and cathode. Also, in a passive DMFC, the anode suffers from high activation overpotentials due to the slow kinetics of methanol oxidation reaction. This negative effect limits the rate of electrode reactions, which reduces the cell voltage and severely affects the voltage efficiency of the system.

The most important challenges to overcome in passive systems are the methanol crossover rate and the heat and water management. Some work has been done in order to evaluate the effect of methanol concentration, methanol crossover and the heat and water management on the cell performance (Cao et al. 2010, Yuan et al. 2011 and 2012, Cai et al. 2011, Zheng 2012). High concentrations of methanol provides a higher achievable energy density, but it also causes severe methanol crossover through the membrane and results in a mixed potential at the cathode, therefore, a lower cell performance (Tan et al. 2010, Yuan 2013, Yousefi and Ganji 2012). The methanol crossover has two negative consequences: self-discharge of methanol, which provides additional heat instead of electricity and drastic reduction of the cathode voltage. Another important factor is the temperature rise in the cell due to the methanol oxidation at the cathode since it releases heat. Moreover, the actual practical electrochemical energy recoverable from a passive DMFC system is much lower than the theoretical value, since only a small percentage of the total energy involved can be converted into electricity, while the rest is lost as heat. This heat has to be dissipated, or else the accumulated heat in the system will rise the temperature of the cell and might become too high for compact portable systems.

The passive feed systems, also, have lower power densities due to the inability to handle the excess of water at the cathode due to the cathode reaction and the water crossed over from the anode. So, a very important engineering issue is to remove water from the cathode to avoid severe flooding and subsequently supply water to the anode to make-up for the water loss due to water crossover. Some studies, regarding the effect of operation and design conditions on the water management, for a passive feed DMFC, have been performed, as the works of Faghri and co-authors (2010 and 2011), Wu, Zhang and Yang (2010) and Oliveira et al. (2013), which allows to understand some of the mechanism involved in water transport and increase the cell power density.

In DMFC carbon dioxide $\left(\mathrm{CO}_{2}\right)$ is produced on the anode leading to a two-phase flow pattern. However, if these bubbles were not removed efficiently from the electrode structure, they can block methanol from reaching the catalyst layer and decrease the methanol oxidation rate and consequently the cell performance. So, understanding and investigate the multiphase flows and the interactions between the layers on the anode side of a DMFC is crucial to develop air breathing operation. Despite the importance of $\mathrm{CO}_{2}$ removal in determining the passive DMFC performance, there is a lack of work on this issue (see Cao et al. 2012) and no detail design optimization has been reported.

As discussed above, many challenges exist for passive DMFC systems. Low water and methanol crossover and high power densities are essential requirements for a DMFC to be used in portable applications. Thus, it is extremely important to develop new membrane electrode assembly designs intended to achieve all the three goals simultaneously. Extensive parametric studies should be performed to elucidate the effects of material properties, MEA fabrication processes and operating conditions in passive feed systems. It is also crucial to develop new models accounting for the effects of coupled heat and mass transfer, along with the electrochemical reactions, preferably consisting of a simple computer-aided tool to design 
and optimize passive DMFCs (Oliveira, Rangel and Pinto 2011). The necessary formulation of adequate mathematical models is based on the need to reach a deeper understanding of internal processes which cannot be observed directly in experiments.

In this work, a detailed experimental study regarding the effects of methanol concentration, membrane thickness and anode and cathode diffusion layer material on the performance of a passive DMFC is described. Since it is very difficult to measure experimentally the methanol and water crossover rates and since these two parameters have a large influence on fuel cell performance, the effect of the different conditions tested experimentally on the methanol and water crossover are explained under the predictions of a one dimensional model previously developed and validated (Oliveira, Rangel and Pinto 2011). The main goal of this work is to methodically vary commercial materials of the MEA and evaluate their influence on the performance of a passive DMFC.

\section{Experiments}

The experimental fuel cell consists of two acrylic end plates (open on the cathode side and with a reservoir on the anode side), two isolating rubber plates, two gold plated copper connector plates (with 36 holes with a diameter of $6 \mathrm{~mm}$ ), two diffusion layers, two catalyst layers and a membrane (Figure 1).

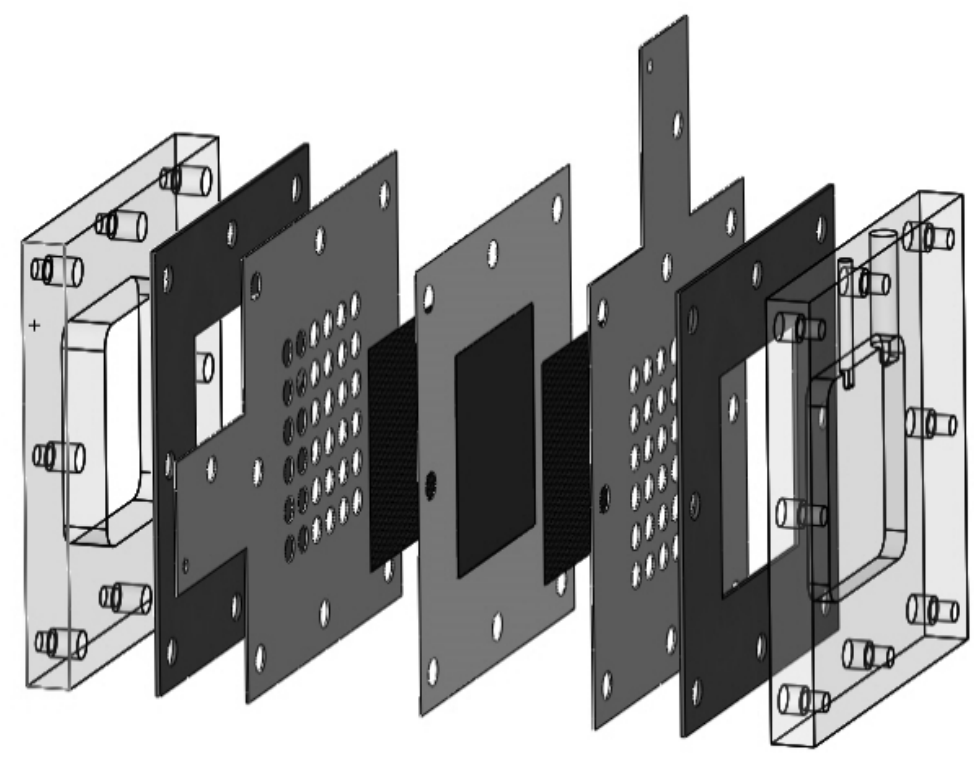

Figure 1: "In-house" passive direct methanol fuel cell

In the experiments, a passive DMFC with a membrane active area of $25 \mathrm{~cm}^{2}$ was operated at atmospheric pressure by feeding aqueous methanol solution to the anode. The fuel cell temperature was controlled by a digital temperature controller and was set near ambient conditions, $20^{\circ} \mathrm{C}$.

Four different methanol concentrations were tested in order to analyze the effect of the methanol concentration on fuel cell voltage and power density.

To evaluate the effect of the membrane thickness on the cell performance, two different membranes with different thicknesses were used, Nafion $117(0.018 \mathrm{~cm})$ and Nafion 115 $(0.0127 \mathrm{~cm})$. The catalyst used on the anode and cathode side was, respectively, $4 \mathrm{mg} / \mathrm{cm}^{2} \mathrm{Pt}-$ $\mathrm{Ru}$ and $4 \mathrm{mg} / \mathrm{cm}^{2} \mathrm{Pt}$.

Since the main goal of the present work is the optimization of a passive DMFC using the materials available commercially, three different materials were selected to be used as both anode and cathode diffusion layers, i.e., carbon cloth (CC), carbon paper (CP) and single-side 
ELAT (ELAT). All three materials are carbon-fiber based porous materials, although carbon paper is non-woven while the other two materials are woven fabric. Regarding their structural parameters, ELAT is the thicker one, carbon cloth the more porous one and carbon paper has the highest tortuosity.

The experimental tests were performed with a commercial fuel cell test station manufactured by Fideris Incorporated (MTK test station) and the polarization curves were performed galvanostatically. A computer constantly monitors both current and voltage and these parameters are used to calculate and track the cell power.

\section{Results and Discussion}

In passive DMFC systems the temperature rises with time due to the electrochemical reactions. In order to minimize this effect on the results presented in this section, all the experiments were conducted at a controlled temperature, ensuring a constant temperature value during each test. The performance of the passive DMFC was determined by a set of tests, in order to obtain the cell polarization and power density curves. The influence of methanol concentration, membrane thickness and anode and cathode diffusion layer material was investigated.

As already mentioned, in a previous work Oliveira, Rangel and Pinto (2011) developed a onedimensional model considering the effects of coupled heat and mass transfer, along with the electrochemical reactions occurring in a passive DMFC. The model is a useful tool to predict the influence of the different operating and design parameters on fuel cell performance and to estimate the methanol crossover rate and the water flux through the membrane, quantified in terms of a net water transport coefficient $(\alpha)$. The ideal net water transport coefficient value is a negative value meaning that the water transport is from the cathode to the anode. The model was validated with data from experiments performed by the authors and with recent published data (Oliveira, Rangel and Pinto 2011).

In this work, model predictions of the influence of the different parameters studied on methanol and water crossover are presented and used to explain the experimental results. The conditions used in the simulations are similar to the ones used in the experiments.

\subsection{Effect of methanol concentration}

In order to evaluate the effect of methanol concentration on fuel cell performance, the cell was operated with four different methanol concentrations ( $1 M, 3 M, 4 M$ and $5 M$ ). The membrane used was Nafion 117, carbon paper and carbon cloth were used, respectively, as anode and cathode diffusion layers. The results regarding the influence of methanol concentration on cell voltage and power density can be found in Figure 2. 

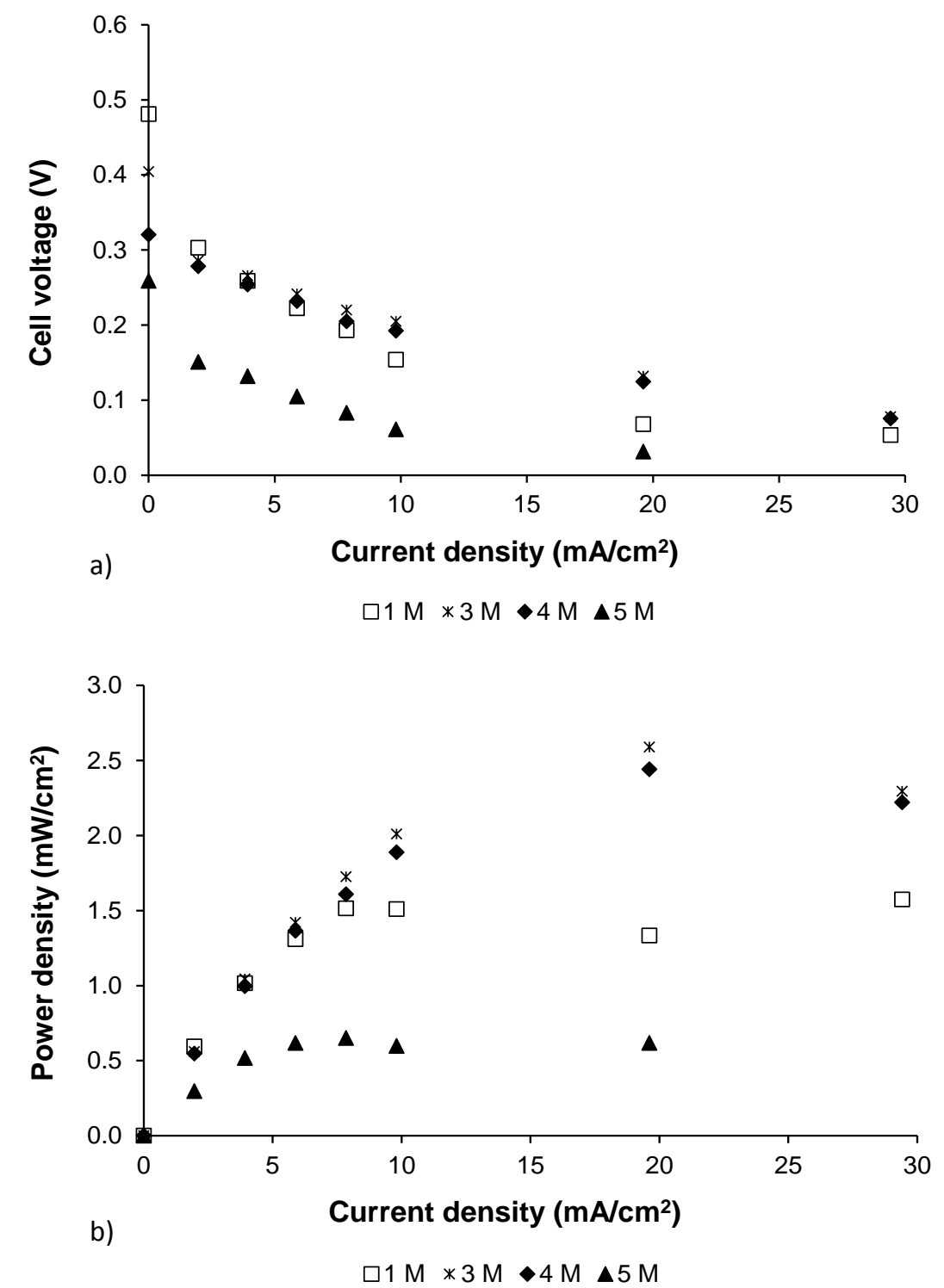

Figure 2. Effect of methanol concentration on a) cell performance and b) power density: experimental results. Design parameters: Nafion 117, carbon paper as anode diffusion layer and carbon cloth as cathode diffusion layer

Two things can be seen on the plots: the open circuit voltage (1) is much lower than the thermodynamic value and (2) decreases with higher methanol concentrations. The latter is due to the fact that higher methanol concentrations have higher methanol crossover rates (Figure 3). Although methanol crossover increases with an increase of methanol concentration, for higher current densities the best performances are achieved with concentrations between $3 \mathrm{M}$ and $4 \mathrm{M}$. Probably due to higher methanol oxidation rate on the anode side, leading to a decrease on the anode overpotential and an overall increase of performance.

Model predictions of the methanol crossover rate for the four methanol feed concentrations tested are presented in Figure 3. As can be seen the methanol crossover increases with an increase of methanol concentration due to a higher concentration gradient between the anode and the cathode side. However, the rate of methanol that crosses the membrane decreases with the current density, as a result of the lower methanol concentrations at the electrode/membrane interface due to higher methanol oxidation rate. 


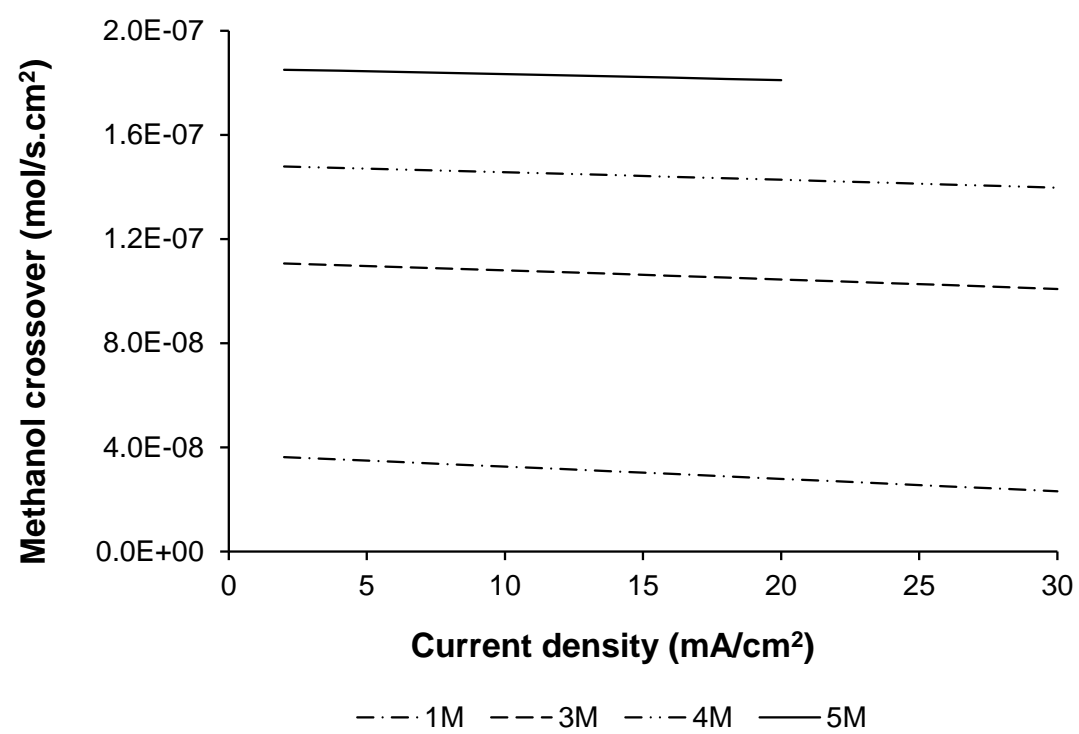

Figure 3: Model predictions for the effect of methanol concentration on methanol crossover. Design parameters: Nafion 117, carbon paper as anode diffusion layer and carbon cloth as cathode diffusion layer Figure 4 shows model predictions of the effect of methanol concentration on the net water transport coefficient. It is evident that the methanol concentration has an impact on the water transport through the membrane. As expected, lower methanol concentrations have higher water transport rates. In such conditions more water is presented on the anode side leading to a higher concentration gradient between the anode and cathode side. Therefore, the transport of water towards the cathode is dominant.

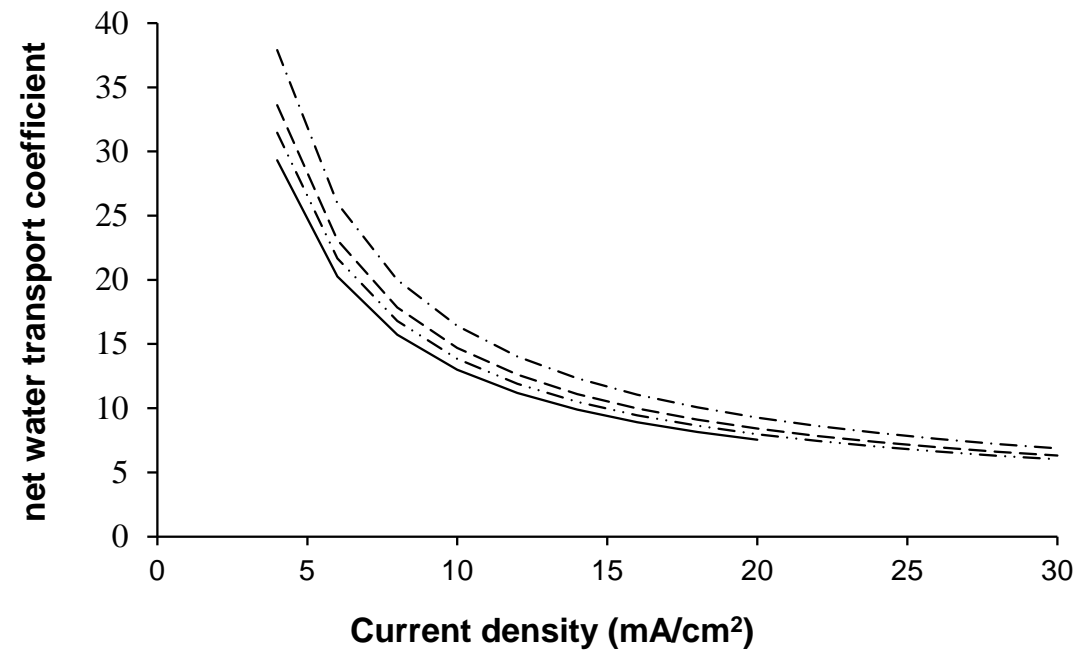

$-\cdot 1 \mathrm{M}---3 \mathrm{M}-\cdot-4 \mathrm{M}-5 \mathrm{M}$

Figure 4: Model predictions for the effect of methanol concentration on net water transport coefficient. Design parameters: Nafion 117, carbon paper as anode diffusion layer and carbon cloth as cathode diffusion layer

\subsection{Effect of membrane thickness}

To study the effect of the membrane thickness on passive fuel cell performance, two different membranes with different thicknesses, Nafion 117 and Nafion 115, were used. The experimental results are shown in Figure 5 for three different methanol concentrations, $1 \mathrm{M}$, $3 \mathrm{M}$ and $5 \mathrm{M}$. It should be mentioned that the methanol and water transport through the membrane are enhanced or diminished by using different membrane thicknesses. The model predictions of the methanol crossover rate and the net water transport coefficient for the two 
membranes tested are presented, respectively in Figure 6 and 7. As can be seen in Figure 5, for all the methanol feed concentrations studied, thicker membranes have higher performances due to lower methanol crossover rates (Figure 6).

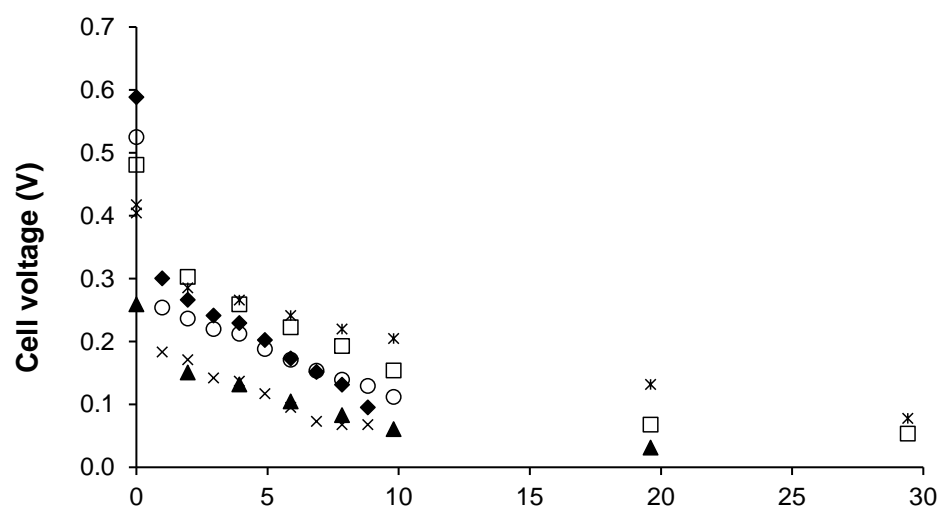

a)

Current density $\left(\mathrm{mA} / \mathrm{cm}^{2}\right)$
$\square 1$ M Nafion 117
*3 M Nafion 117
\5 M Nafion 117
• 1 M Nafion 115
○3 M Nafion 115
$\times 5$ M Nafion 115

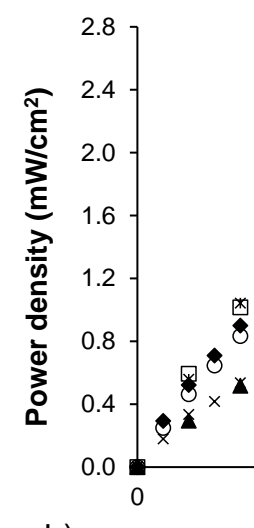

b)

Current density $\left(\mathrm{mA} / \mathrm{cm}^{2}\right)$
$\square 1$ M Nafion 117
*3 M Nafion 117
\5 M Nafion 117
-1 M Nafion 115
O3 M Nafion 115
$\times 5$ M Nafion 115

Figure 5: Effect of membrane thickness on a) cell performance and b) power density, for three different methanol feed concentrations ( $1 \mathrm{M}, 3 \mathrm{M}$ and $5 \mathrm{M}$ ): experimental results. Design parameters: carbon paper as anode diffusion layer and carbon cloth as cathode diffusion layer

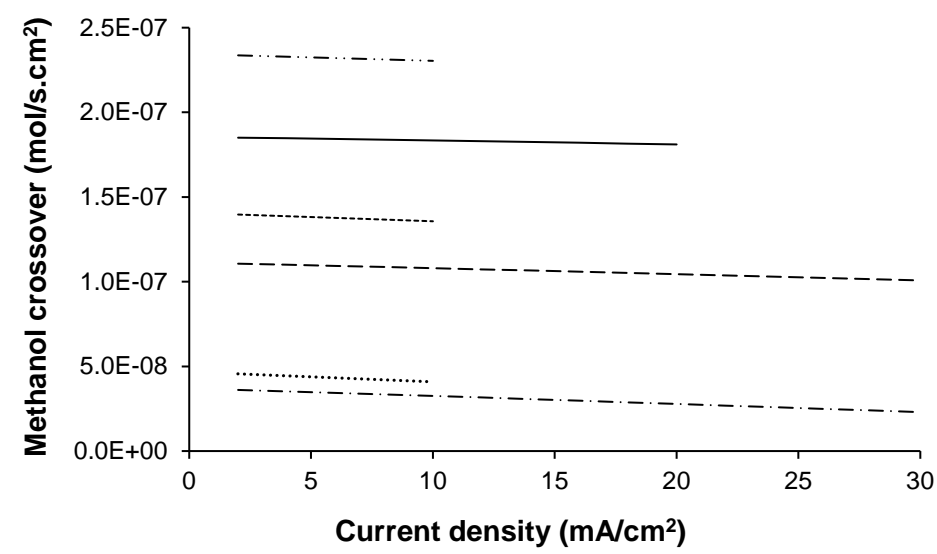

$$
\begin{aligned}
& \text {-. } 1 \mathrm{M} \text { Nafion } 117---3 \mathrm{M} \text { Nafion } 117-5 \mathrm{M} \text { Nafion } 117 \\
& \text {.........1M Nafion } 115 \text {-.-.--3M Nafion } 115 \text {-..-5M Nafion } 115
\end{aligned}
$$

Figure 6: Model predictions for the effect of membrane thickness on methanol crossover, for three different methanol feed concentrations (1 M, $3 \mathrm{M}$ and $5 \mathrm{M}$ ). Design parameters: carbon paper as anode diffusion layer and carbon cloth as cathode diffusion layer 
The predicted values for the net water transport coefficient for the two membranes tested are presented in Figure 7. As can be seen, decreasing the membrane thickness enhances the water back transport, from the anode to the cathode, a very important condition for operating a fuel cell with high methanol concentrations or even pure methanol. Also, thinner membranes have lower costs and lower ohmic resistances. Based on that, it would be advantageous to use thinner membranes and limit the amount of methanol that crosses the membrane using thicker diffusion and catalyst layers (Oliveira et al. 2013).

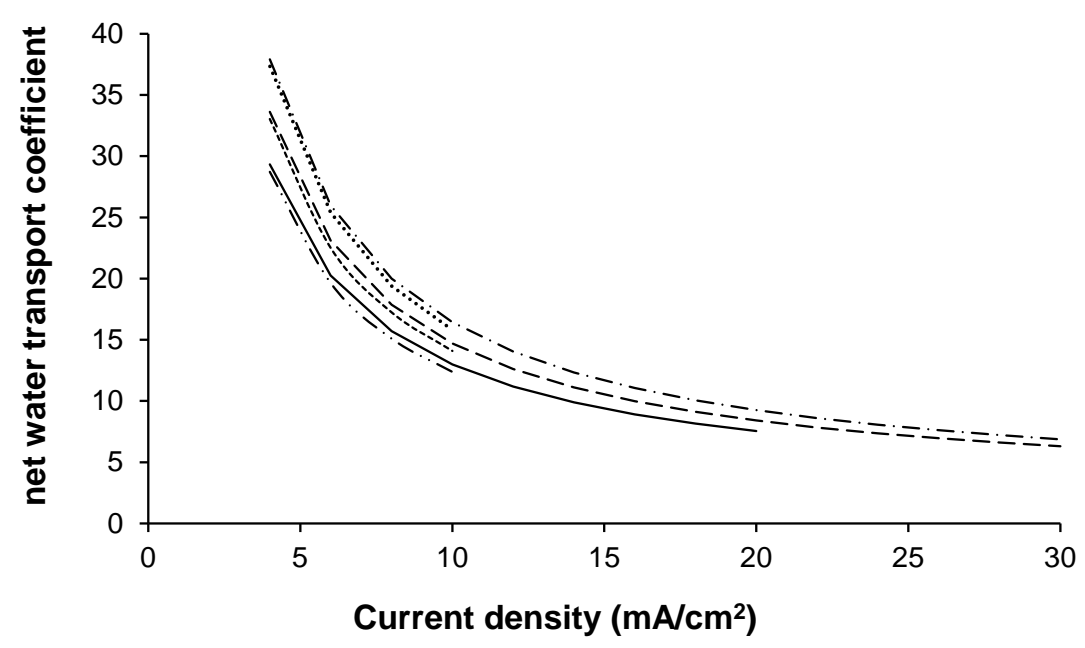

$$
\begin{aligned}
& \text {-. -.1M Nafion } 117 \text { - - - 3M Nafion } 117 \_ \text {5M Nafion } 117 \\
& \text {........ 1M Nafion } 115 \text {---.--3M Nafion } 115 \text {-.-5M Nafion } 115
\end{aligned}
$$

Figure 7: Model predictions for the effect of membrane thickness on net water transport coefficient, for three different methanol feed concentrations (1 M, $3 \mathrm{M}$ and $5 \mathrm{M}$ ). Design parameters: carbon paper as anode diffusion layer and carbon cloth as cathode diffusion layer

\subsection{Effect of anode diffusion layer}

Several materials and design parameters of the diffusion layers may affect the fuel cell performance, such as layer thickness and tortuosity, which influences the species transport and the surface properties, wettability and roughness. Differences in these properties result in different transport characteristics. As mentioned, in order to evaluate the effect of the anode diffusion layer material on fuel cell voltage and power density, three different materials were used, carbon cloth (CC), carbon paper (CP) and ELAT.

The influence of the anode diffusion layer on the cell voltage and power density are shown in Figure 8. The experimental results indicate that better performances are achieved using ELAT as anode diffusion layer, mostly due to its higher thickness when compared to the other two materials used. Thicker diffusion layers have higher mass transport resistances, controlling the amount of methanol that crosses the membrane (Figure 9). As the cell performance is strongly dependent on methanol crossover, higher performances are obtained. However, since ELAT and carbon cloth have similar thicknesses, the difference on thickness may not be the only reason for the differences in performance. These may be explained by the fact that ELAT is a carbon cloth electrode treated with Platinum on the side that is in contact with the catalyst layer, having for this reason an extra catalyst loading. In such conditions, more active sites are presented for methanol oxidation reaction, decreasing the anode overpotential and increasing the fuel cell performance. Due to its properties, the cell using the ELAT material can be operated with higher methanol concentrations $(5 \mathrm{M})$ without sacrificing the performance. 

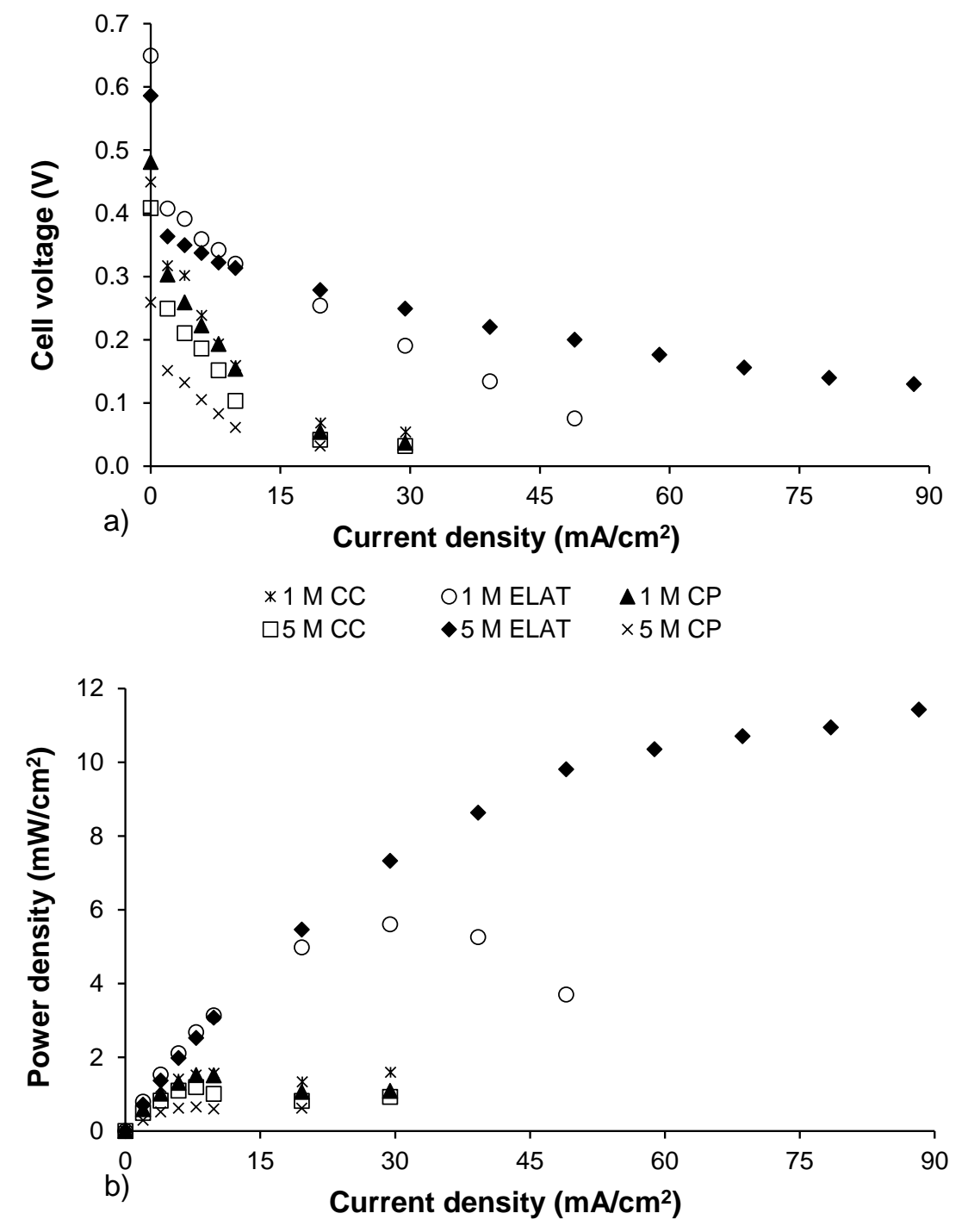

$$
\begin{array}{lll}
* 1 \mathrm{M} \mathrm{CC} & \text { O1 M ELAT } & \Delta 1 \mathrm{M} \mathrm{CP} \\
\square 5 \mathrm{M} \mathrm{CC} & \diamond 5 \mathrm{M} \mathrm{ELAT} & \times 5 \mathrm{M} \mathrm{CP}
\end{array}
$$

Figure 8: Effect of anode diffusion layer material on a) cell performance and $b$ ) power density, for two different methanol feed concentrations ( $1 \mathrm{M}$ and $5 \mathrm{M}$ ): experimental results. Design parameters: Nafion 117 and carbon cloth as cathode diffusion layer

It should, also, be mentioned, that on the anode side gaseous carbon dioxide is produced by the anode reaction which must be efficiently removed from the catalyst layer. If the carbon dioxide bubbles cannot be removed from the catalyst surface, they cover the surface decreasing the effective active area for methanol oxidation. The texture of carbon paper with a highly tortuous structure enhances the interactions between the bubbles and the solid. Hence the gas tends to remain attached to the surface leading to a blockage of the active sites lowering the methanol oxidation reaction rate and the cell performance. 


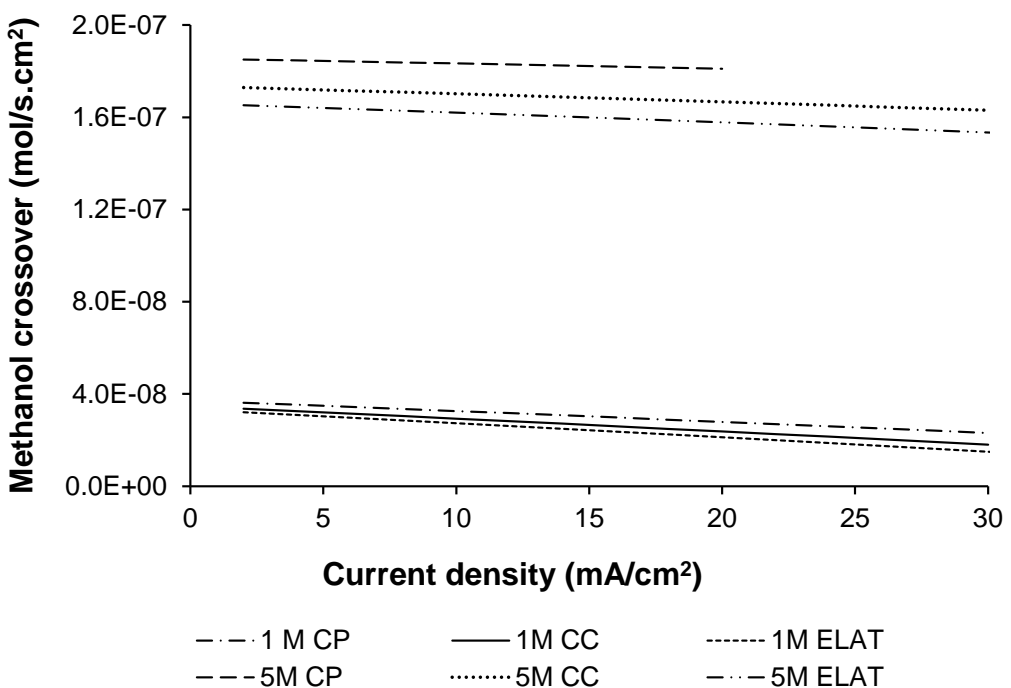

Figure 9: Model predictions for the effect of anode diffusion layer material on methanol crossover, for two different methanol feed concentrations ( $1 \mathrm{M}$ and 5 M). Design parameters: Nafion 117 and carbon cloth as cathode diffusion layer

Figure 10 shows the effect of the anode diffusion layer material on the net water transport coefficient. The plots show that thicker anode diffusion layers have higher mass transport resistances, hence reducing the amount of water that reaches the membrane and consequently the water flux towards the cathode. So thicker anodes have lower net water transport coefficients. These results are in accordance to the ones presented above regarding the methanol crossover through the membrane.

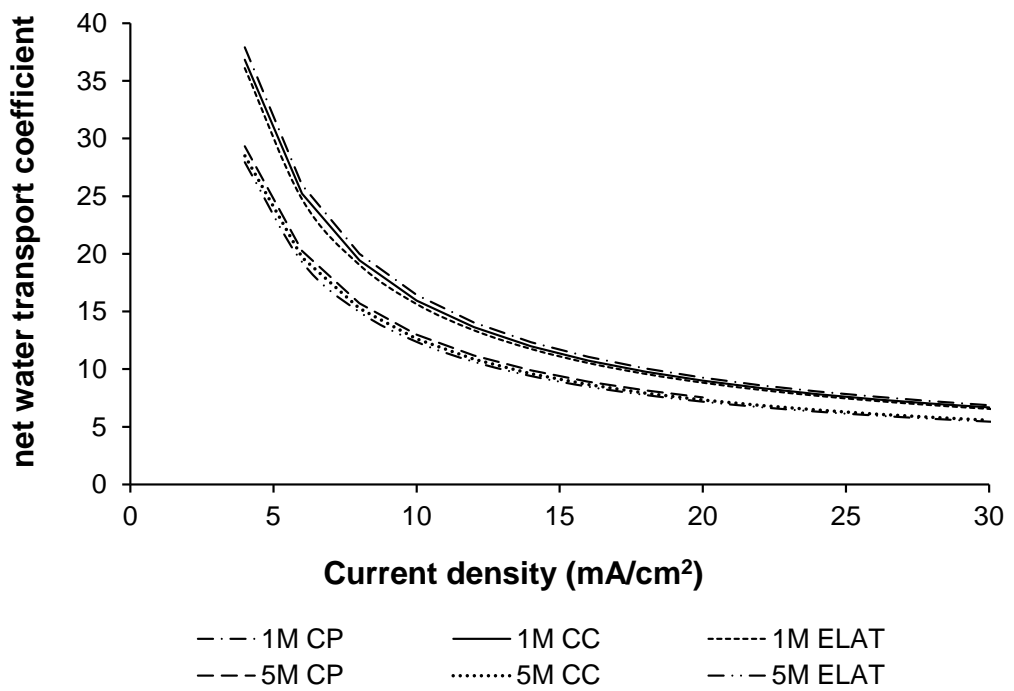

Figure 10: Model predictions for the effect of anode diffusion layer material on net water transport coefficient, for two different methanol feed concentrations ( $1 \mathrm{M}$ and $5 \mathrm{M}$ ). Design parameters: Nafion 117 and carbon cloth as cathode diffusion layer

\subsection{Effect of cathode diffusion layer}

As in the previous section, three different materials were used to study the effect of the diffusion layer material on fuel cell performance: CC, CP and ELAT, but now on the cathode. The results are presented in Figure 11. It should be noted that the differences in performance with different cathode diffusion media are due to (i) the cell's internal resistances, (ii) the oxygen transport and (iii) water removal. As can be seen from Figure 11, the lowest performance is achieved using CP as cathode diffusion layer. Since CP is highly tortuous, it 
increases the interactions between the water and the surface of the gas diffusion layers (GDL). Therefore the water tends to remain attached to the surface, leading to a blockage of the active sites with a consequent decrease of the oxygen reduction reaction rate and cell performance. Also, when compared to the other two materials tested, carbon paper with its high tortuosity, imposes a higher oxygen transport resistance with lower oxygen concentrations in the surface of the catalyst layer.

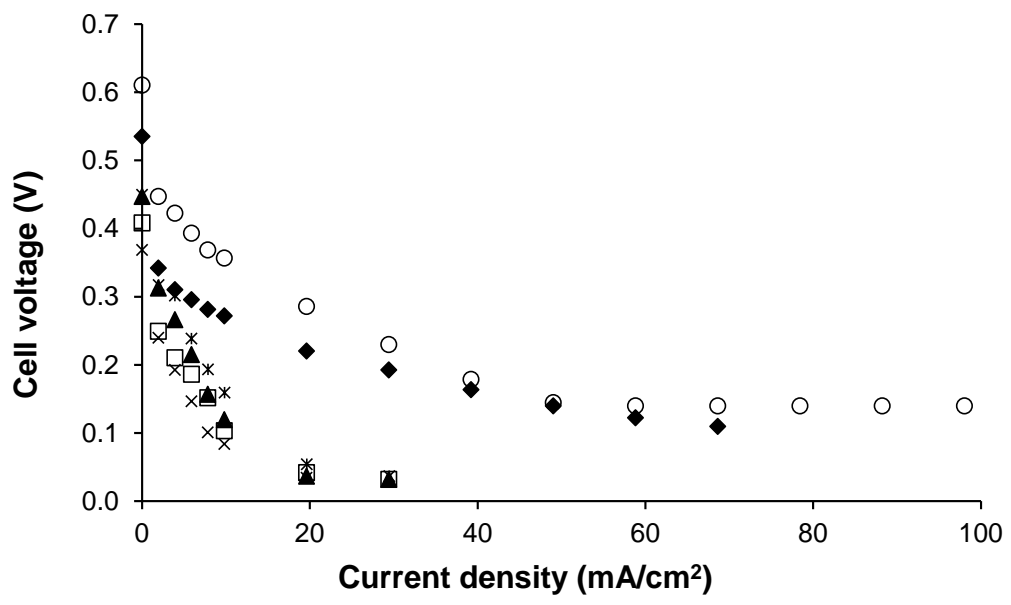

a)

$$
\begin{array}{lll}
* 1 \mathrm{M} \mathrm{CC} & \text { 01 M ELAT } & \boldsymbol{\Delta 1 M ~ M ~ C P} \\
\square 5 \mathrm{M} \mathrm{CC} & \diamond 5 \mathrm{M} \mathrm{ELAT} & \times 5 \mathrm{M} \mathrm{CP}
\end{array}
$$

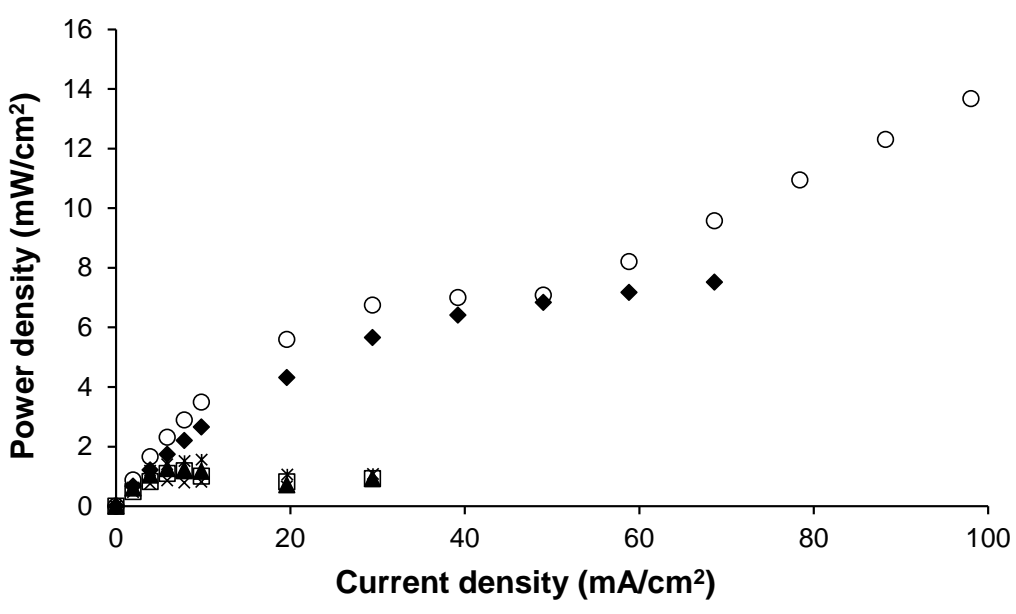

b)

$$
\begin{array}{lll}
* 1 \mathrm{M} \mathrm{CC} & \text { O1 M ELAT } & \Delta 1 \mathrm{M} \mathrm{CP} \\
\square 5 \mathrm{M} \mathrm{CC} & 5 \mathrm{M} \mathrm{ELAT} & \times 5 \mathrm{M} \mathrm{CP}
\end{array}
$$

Figure 11: Effect of cathode diffusion layer material on a) cell performance and b) power density, for two different methanol feed concentrations (1 $M$ and $5 \mathrm{M})$ : experimental results. Design parameters: Nafion 117 and carbon cloth as anode diffusion layer

Using ELAT as cathode diffusion layer leads to higher performances, mainly due to its treatment with Platinum, which increases the amount of active sites for oxygen reduction reaction and consequently decreases the cathode overpotential. However, contrarily to the previous analyzed case, the highest performance is achieved with low methanol concentrations (see Figure 11). This is due to the fact that lower methanol concentrations have lower methanol crossover rates (Figure 12), which is very advantageous for decreasing the cathode overpotential, since the mixed potential formation due to methanol oxidation reaction on the cathode side may be avoided in some extent. Less catalyst sites are used by the permeated methanol, so more active sites are free for oxygen reaction. 


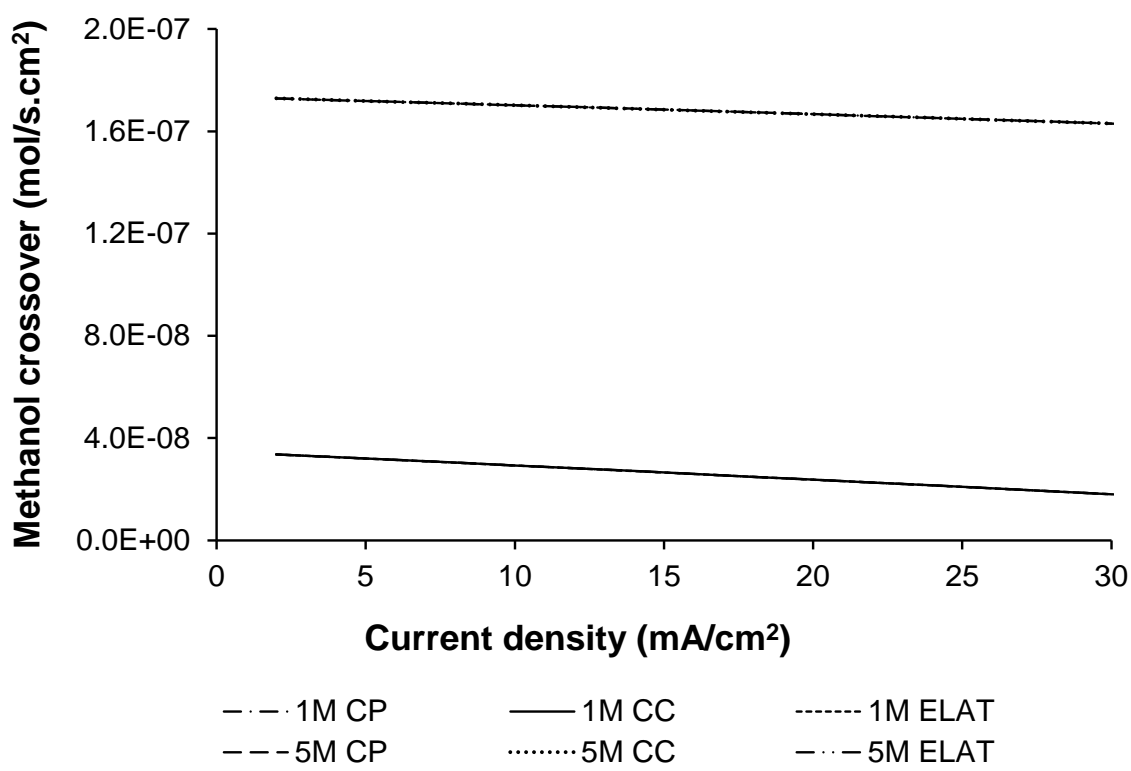

Figure 12: Model predictions for the effect of cathode diffusion layer material on methanol crossover, for two different methanol feed concentrations ( $1 \mathrm{M}$ and 5 M). Design parameters: Nafion 117 and carbon cloth as anode diffusion layer

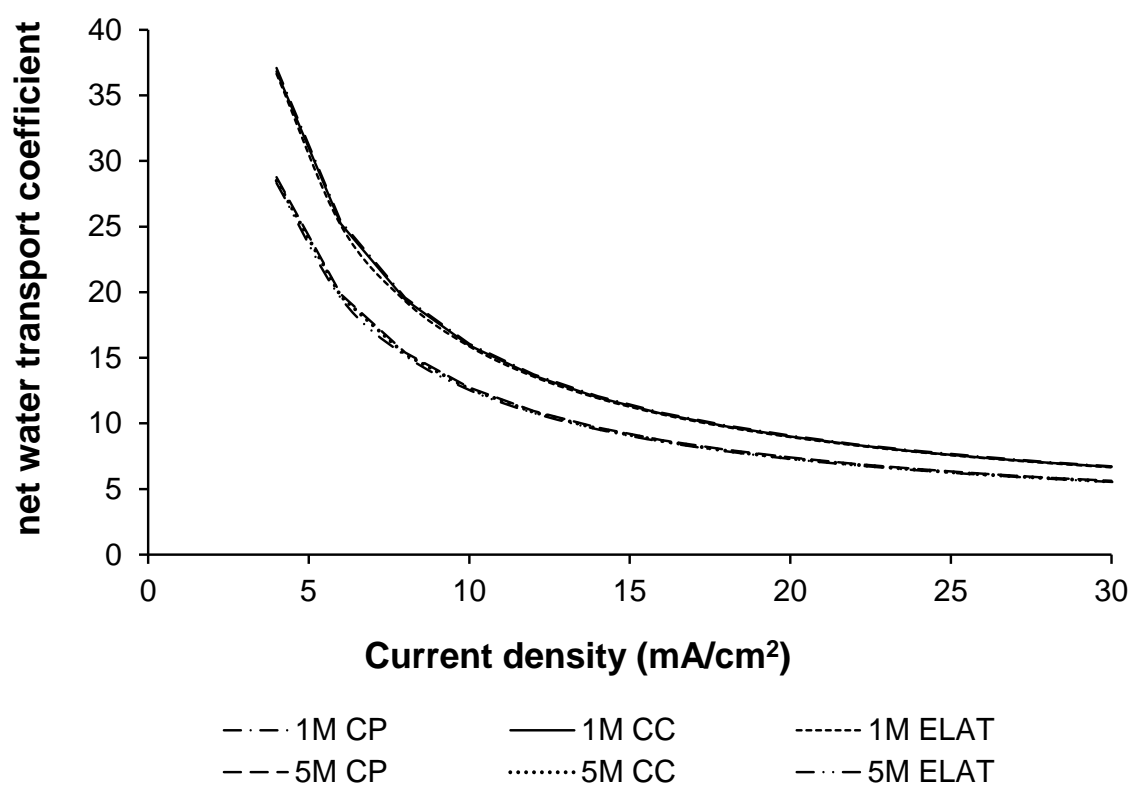

Figure 13: Model predictions for the effect of cathode diffusion layer material on net water transport coefficient, for two different methanol feed concentrations ( $1 \mathrm{M}$ and $5 \mathrm{M}$ ). Design parameters: Nafion 117 and carbon cloth as anode diffusion layer

Model predictions for the effect of cathode diffusion layer on methanol crossover and net water transport coefficient are presented in Figure 12 and Figure 13. The results from both figures show that for the studied conditions, the cathode diffusion layer material has a negligible effect on methanol and water transport through the membrane from the anode to the cathode side. These results show that the main influence of the cathode material on fuel cell performance is related to (i) the oxygen transport, (ii) oxygen reaction rate and (iii) water removal from the cathode.

\section{Conclusions}

A great challenge on the development of passive DMFC, in order to be used in real applications, is to use cost-effective materials and cells with high energy densities and low 
methanol and water crossover rates. To achieve that it is necessary to use high methanol concentrations and use different membrane, diffusion layers and catalyst layers materials with different thicknesses. Based on that, this work aims to solve some of these problems and will certainly contribute to an advance on the DMFC knowledge for portable applications.

Therefore, in this work, the effect of methanol concentration, membrane thickness and anode and cathode diffusion layer material on fuel cell performance is presented. The results are explained by predictions of the methanol crossover rate and the net water transport coefficient from a developed and validated model. Based on that, the model simulations regarding the effect of the different parameters studied experimentally on methanol and water crossover were also included.

Very high methanol concentrations achieve lower fuel cell performances due to higher methanol crossover rates. However, lower methanol concentrations have higher net water transport coefficients, so more water crosses the membrane from the anode to the cathode side.

Increasing the membrane and the anode diffusion layer thickness, significantly increases the fuel cell performance due to a decrease of the methanol crossover. Also, thicker anode diffusion layers lead to lower net water transport coefficients.

In this work the maximum power density, $13.7 \mathrm{~mW} / \mathrm{cm}^{2}$, was obtained using a Nafion 117 membrane, $4 \mathrm{mg} / \mathrm{cm}^{2}$ of Pt-Ru and $4 \mathrm{mg} / \mathrm{cm}^{2}$ of Pt, as respectively, anode and cathode catalyst layers, carbon cloth as anode diffusion layer, ELAT as cathode diffusion layer and a methanol concentration of $1 \mathrm{M}$. However, a comparable power density, $11.4 \mathrm{~mW} / \mathrm{cm}^{2}$, was obtained using a higher methanol concentration, $5 \mathrm{M}$, with the same membrane and anode and cathode catalyst loadings, but using ELAT as anode diffusion layer and carbon cloth as cathode diffusion layer. As a result of this work, a tailored MEA build-up with the common available commercial materials was proposed to achieve relatively low methanol and water crossover, operating at high methanol concentrations.

The obtained results, also, show that changes in fuel cell structure are effective ways of controlling the methanol and water crossover and increasing the fuel cell performance and provide a basic element for future passive DMFC systems working with high methanol concentrations or pure methanol.

Further improvements will be achieved when more structured diffusion layers and more cost effective materials for the different layers become commercially available.

It is expected, that this work, will induce the consumers to believe and accept fuel cells as an emerging technology.

\section{References}

Bahrami, Hafez and Amir Faghri A. "Water management in a passive DMFC using highly concentrated methanol solution." Journal of Fuel Cell Science and Technology 8 (2011b): 115. DOI: 10.1115/1.4002315.

Barbir, Frano. 2005. PEM Fuel Cells: Theory and Practice. USA: Elsevier Academic Press. ISBN: 978-0-12-078142-3.

Cai, Weiwei, Songtao Li, Liang Yan, Ligang Feng, Jing Zhang, Liang, Wei Xing and Changpeng Liu." Design and simulation of a liquid electrolyte passive direct methanol fuel cell with low methanol crossover." Journal of Power Sources 196 (2011):7616-7626. DOI: 10.1016/j.jpowsour.2011.05.006. 
Cao, Jianyu, Mei Chen, Ji Chen, Shenjun Wang, Zhiqing Zou, Zhilin Li, Daniel L. Akins and Hui Yang. "Double microporous layer cathode for membrane electrode assembly of passive direct methanol fuel cells." International Journal of Hydrogen Energy 35 (2010):4622-4629. DOI: 10.1016/j.ijhydene.2010.02.012.

Cao, Xian Q., Ji T. Han, Ze T. Yu, Peng C. Dou, Pei P. Chen. "Visualization study of $\mathrm{CO}_{2}$ bubble behavior in passive direct methanol fuel cell." Advanced Materials Research 457-458 (2012):98-101. DOI: 10.4028/www.scientific.net/AMR.457-458.98.

$\mathrm{Li}$, Xianglin, Amir Faghri and Chao Xu. "Water management of the DMFC passively fed with a high-concentration methanol solution." International Journal of Hydrogen Energy 35 (2010):8690-8698. DOI: 10.1016/j.ijhydene.2010.05.033.

Oliveira, V. B., C. M. Rangel, and A. M. F. R. Pinto. "One-dimensional and non-isothermal model for a passive DMFC." Journal of Power Sources 196 (2011):8973-8982. DOI: 10.1016/j.jpowsour.2011.01.094.

Oliveira, Vânia B., Daniela S. Falcão, Carmen M. Rangel and Alexandra M. F. R. Pinto. "Water management in a passive direct methanol fuel cell." International Journal of Energy Research 37 (2013): 991-1001. DOI: 10.1002/er.2902.

Pan, Y.H. "Advanced air-breathing direct methanol fuel cells for portable applications." Journal of Power Sources 161 (2006):282-289. DOI: 10.1016/j.powsour.2006.03.048.

Sundmacher, Kai. "Fuel cell engineering: toward the design of efficient electrochemical power plants." Ind. Eng. Chem. Res. 49 (2010):10159-10182. DOI: 10.1021/ie100902t.

Tang, Yong, Wei Yuan, Minqiang Pan, Biao Tang, Zongtao Li and Zhenping Wan. "Effects of structural aspects on the performance of a passive air-breathing direct methanol fuel cell." Journal of Power Sources 195 (2010):5628-5636. DOI: 10.1016/j.jpowsour.2010.03.069.

Wu, Q.X., T. S. Zhao and W. W. Yang. "Effect of the cathode gas diffusion layer on the water transport behavior and the performance of passive direct methanol fuel cells operating with neat methanol." International Journal Heat and Mass Transfer 54 (2011):1132-1143. DOI: 10.1016/j.ijheatmasstransfer.2010.11.009.

$\mathrm{Xu}$, Chao and Amir Faghri. "Water transport characteristics in a passive liquid-feed DMFC." International Journal Heat and Mass Transfer 53 (2010b):1951-1966. DOI: 10.1016/j.ijheatmasstransfer.2009.12.060.

Yousefi, Sasan and Davood D. Ganji. "Experimental investigation of a passive direct methanol fuel cells with $100 \mathrm{~cm}^{2}$ active areas." Electrochimica Acta 85 (2012):693-699. DOI: 10.1016/j.electacta.2012.08.045.

Yuan, W., Y. Tang, X. Yang and Z. Wan. "Toward fully-and semi-passive operation of a liquidfed direct methanol fuel cell." Fuel Cells 13 (2013):249-258. DOI: 10.1002/fuce.201200145.

Yuan, Wei, Yong Tang, Xiaojun Yang, Bin Liu and Zhenping Wan. "Structural diversity and orientation dependence of a liquid-fed passive air-breathing direct methanol fuel cell." International Journal of Hydrogen Energy 37 (2012):9298-9313. DOI: 10.1016/j.ijhydene.2012.03.013.

Yuan, Wei, Yong Tang, Zhenping Wan and Minqiang Pan. "Operational characteristics of a passive air-breathing direct methanol fuel cell under various structural conditions." International Journal of Hydrogen Energy 36 (2011):2237-2249. DOI: 10.1016/j.ijhydene.2010.11.067. 
Zheng, Wukui, Arho Suominen, Jarno Kankaanranta and Aulis Tuominen. "A new structure of a passive direct methanol fuel cell." Chemical Engineering Science 76 (2012):188-191. DOI: 10.1016/j.ces.2012.04.028.

\section{Acknowledgements}

Beatriz A. Berns acknowledges the Ph.D. fellowship (BEX 12997/13-7) supported by CAPES Foundation, Ministry of Education of Brazil. Vânia B. Oliveira acknowledges the post-doctoral fellowship (SFRH/BDP/91993/2012) supported by the Portuguese "Fundação para a Ciência e Tecnologia" (FCT), POPH/QREN and European Social Fund (ESF). POCI (FEDER) also supported this work via CEFT. 